\title{
STIMULATIONS AUDITIVES CONTROLATÉRALES ET MÉCANISMES ACTIFS COCHLÉAIRES : VULNÉRABILITÉ PHYSIOLOGIQUE DE LA COCHLÉE
}

\author{
E. VEUILLET, L. COLLET et A. MORGON \\ URA CNRS 1447, Physiologie Sensorielle "Audition et Voix", Hôpital Edouard Herriot, 3 place \\ d'Arsonvah, F-69437 Lyon Cedex 03, France
}

\begin{abstract}
RESUME: Les otoémissions acoustiques provoquées (OEAP) ont été enregistrées en présence de stimulations auditives controlatérales afin d'étudier l'effet suppresseur sur les différentes composantes spectrales de la réponse. Les résultats montrent qu'alors que l'amplitude de certaines bandes de fréquence diminue particulièrement bien et de façon fréquence spécifique, d'autres ne montrent que de faibles diminutions, non liées à la fréquence. Comme l'effet suppresseur semble inefficace sur les fréquences de l'OEAP aux alentours de 4 $\mathrm{kHz}$, un rôle protecteur du système efférent médian est discuté.
\end{abstract}

\begin{abstract}
The evoked otoacoustic emissions (OEAP) were recorded in presence of contralateral acoustic stimulations in order to study the suppressive effect on the different frequency components of the response. The results show that the amplitude of some frequency bands decreases in a frequency selectivity manner, but for other bands, the decrease is small without frequency specificity or even absent. This absence of suppressive effect is more particularly observed on the frequency bands around $4 \mathrm{kHz}$. So, a protective role of the medial efferent system is discussed.
\end{abstract}

\section{INTRODUCTION}

Des études effectuées chez l'humain (1) et chez l'animal (2) ont mis en évidence que les otoémissions acoustiques (OEA), (sons supposés traduire les mécanismes cochléaires actifs des cellules ciliées externes (CCE) de l'Organe de Corti (3)), peuvent être modifiées en présence de stimulations auditives controlatêrales: l'effet va dans le sens d'une diminution de l'amplitude des OEA. Anatomiquement, on sait que sur les CCE viennent se projeter des fibres appartenant au système efférent médian (4) et il est donc possible que l'effet suppresseur controlatéral emprunte cette voie. D'autant plus que sa présence chez des sujets sans réflexe stapédien $(1,5)$ ou chez des cobayes dont les tendons du muscle stapédien ont été sectionnés, et surtout sa bonne spécificité fréquentielle sont de puissants arguments contre l'implication exclusive de l'oreille moyenne. Chez l'animal, il a pu en outre être montré que l'effet disparaissait après section des fibres efférentes au niveau du plancher du 4 ème ventricule (2).

Pour mieux connaître les modalités d'efficacité de la stimulation auditive controlatérale, nous avons entrepris une série d'études dont sont issus les quelques résultats présentés ici. 


\section{METHODES EXPERIMENTALES}

Les études ont été conduites chez l'humain. Elles ont consisté à enregistrer les otoémissions acoustiques provoquées (OEAP) en l'absence puis en présence de stimulations auditives controlatérales et d'en étudier l'effet sur les composantes spectrales de la réponse.

\subsection{Procédure}

Pour l'enregistrement des OEAP, une sonde se composant d'un écouteur et d'un microphone est placée dans le conduit auditif externe du sujet (oreille qualifiée d'ipsilatérale). Le logiciel ILO88 Otodynamic, mis au point par BRAY and KEMP (6), a été utilisé pour le recueil et l'analyse des données. Un écouteur, placé sur l'autre oreille permet d'administrer la stimulation controlatérale générée par un audiomètre.

Chaque procédure expérimentale est détaillée dans le tableau ci-dessous:

Protocoles expérimentaux observés durant chaque étude: A chaque condition de stimulation ipsilatérale correspond une condition sans et une condition avec stimulation controlatérale. CNF: click non filtré (bande passante 500-6000 Hz); 5 int.: 5 intensités entre 60 et 72 dBSPL par pas de $3 \mathrm{~dB}$; BB: bruit blanc à large bande; NB: bruits blancs à bande étroite (fréquence centrale $\pm 1 / 6$ d'octave: $0.5,0.75,1,1.5,2,3,4,6 \mathrm{kHz}$ ).

\begin{tabular}{|c|c|c|c|}
\hline & Nombre de sujets & $\begin{array}{c}\text { Stimulation } \\
\text { Ipsilatérale }\end{array}$ & $\begin{array}{c}\text { Stimulation } \\
\text { Controlatérale }\end{array}$ \\
\hline Etude 1 & 11 & $\begin{array}{c}\mathrm{CNF} \\
60 \pm 3 \mathrm{~dB}\end{array}$ & $\begin{array}{c}\mathrm{BB} \\
30 \mathrm{~dB} \text { SL }\end{array}$ \\
\hline Etude 2 & 46 & $\begin{array}{c}\mathrm{CNF} \\
5 \mathrm{intensités}\end{array}$ & $\begin{array}{c}\mathrm{BB} \\
\mathbf{d B} \mathrm{dL}\end{array}$ \\
\hline Etude 3 & 16 & $\begin{array}{c}\mathrm{CNF} \\
5 \text { intensités }\end{array}$ & $\begin{array}{c}\mathrm{NB} \\
50 \mathrm{~dB} \text { SPL }\end{array}$ \\
\hline
\end{tabular}

\section{II.2. Analyse des données}

Chaque réponse a été decomposée en 24 bandes de $200 \mathrm{~Hz}$ dont la dénomination correspond à la fréquence centrale. Pour les études 2 et 3 , où 5 intensités de stimulation ont été utilisées, le calcul de l'atténuation équivalente a été effectué selon la procédure détaillée ailleurs (5). $L^{\prime}$ analyse de la variance sur mesures répétées et le test $t$ de Student ont été employés pour le traitement statistique des données.

\section{RESULTATS}

Lors de la première étude, nous avons pu mettre en évidence que chez 6 des 11 sujets présentant des otoémissions sur toutes les bandes de fréquence jusqu'à $5.0 \mathrm{kHz}$, il existait une différence significative de l'effet de la stimulation auditive controlatérale entre les composantes spectrales ( $F 90,5=2.46 ; \mathrm{p}<0.01$ ). Alors qu'un effet statistiquement significatif est observé sur les bandes 0.8 à $1.8 \mathrm{kHz}$, aucun effet significatif existe sur les bandes 2.0 à $4.7 \mathrm{kHz}$; en revanche une diminution significative est à nouveau retrouvée sur la bande correspondant à la fréquence centrale de $5.0 \mathrm{kHz}$.

Lors de la seconde étude, en utilisant une meilleure méthode de quantification de l'effet suppresseur controlatéral et en agrandissant le nombre de sujets, l'absence total d'effet significatif de la stimulation controlatérale se limite à la bande de fréquence centrale égale à $4.2 \mathrm{kHz}$. Toutefois, les amplitudes des bandes 4.0 a $4.4 \mathrm{kHz}$ sont significativement moins affectées par la stimulation controlatérale que celles des bandes 0.8 à $2.0 \mathrm{kHz}$. 
La troisième étude montre que les composantes spectrales de 1'OEAP de 0.5 à $3.0 \mathrm{kHz}$ sont modifiées de façon significativement différentes en fonction de la fréquence du bruit à bande étroite administré dans l'oreille controlatérale. Elles diminuent plus intensément en présence du stimulus présentant la fréquence centrale la plus proche de la leur (Figure 2). Par contre, pour les bandes de fréquence de 3.3 à $5.0 \mathrm{kHz}$, la fréquence du stimulus controlatéral n'a aucune influence sur l'amplitude de la diminution. La figure 2 montre les résultats obtenus sur 2 des 24 bandes de fréquence étudiées ( $O$ fréquence centrale $1 \mathrm{kHz}$; fréquence centrale $4 \mathrm{kHz}$ ).

\section{DISCUSSION}

L'ensemble des résultats obtenus indique que les composantes spectrales de l'OEAP ne sont pas toutes affectées de façon similaire par la stimulation auditive controlatérale; en effet, alors qu'un effet suppresseur existe sur les composantes entre 0.5 et $3.9 \mathrm{kHz}$ et entre 4.6 et $5.3 \mathrm{kHz}$, l'effet est absent sur les bandes entre 4.1 et $4.6 \mathrm{kHz}$. Ils confirment par ailleurs l'existence d'une spécificité fréquentielle de l'effet suppresseur controlatéral déjà démontré dans une précédente étude (5) et qui permet d'éliminer l'intervention exclusive de l'oreille moyenne. La moins bonne efficacité de cet effet sur les fréquences comprises entre 4.1 et $4.6 \mathrm{kHz}$ pourrait être imputable à une moins bonne projection des fibres efférentes sur les CCE impliquées dans la ré-émission des sons à ces fréquences. Or, chez l'animal, les régions cochléaires les plus riches en innervation efférente sont les régions basales (7). Nos résultats seraient donc plutôt en faveur d'une moins bonne efficacité fonctionnelle de ces fibres aux alentours de $4 \mathrm{kHz}$, donnant naissance à une vulnérabilité physiologique de la cochlée pour des fréquences connues pour être les premières atteintes lors d'exposition aux bruits intenses. Ces résultats serait donc en faveur d'un effet protecteur des fibres efférentes sur les mécanismes cochléaires $(8,9)$.

\section{REFERENCES}

/1/COLLET, L., VEUILLET, E., DUCLAUX, R., MOULIN, A. et MORGON, A. Hearing Res 43 (1989) 252).

/2/PUEL, J.L.et REBILLARD, G. J.Acoust.Soc.Am 87 (1990) 1630).

13/KEMP, D.T. J.Acoust.Soc.Am. 64 (1979) 1386).

/4/WARR, W.B.et GUINAN, J.J .Jr. Brain Res 173 (1979) 152).

15/VEUILLET, E., COLLET, L.et DUCLAUX, R. J.Neurophysiol 65 (1991) 724).

16/BRAY, P. et KEMP, D.T. Br.J.Audiol. 21 (1987) 191).

17/LIBERMAN, M.C. J.Comp.Neurol. 301 (1990) 443).

18/RAJAN, R. et JOHNSTONE, B.M. Hearing Res 39 (1989) 263).

19/PATUZZI, R. et THOMPSON M.L. Hearing Res $\underline{54}$ (1991) 45).

\section{FIGURES}



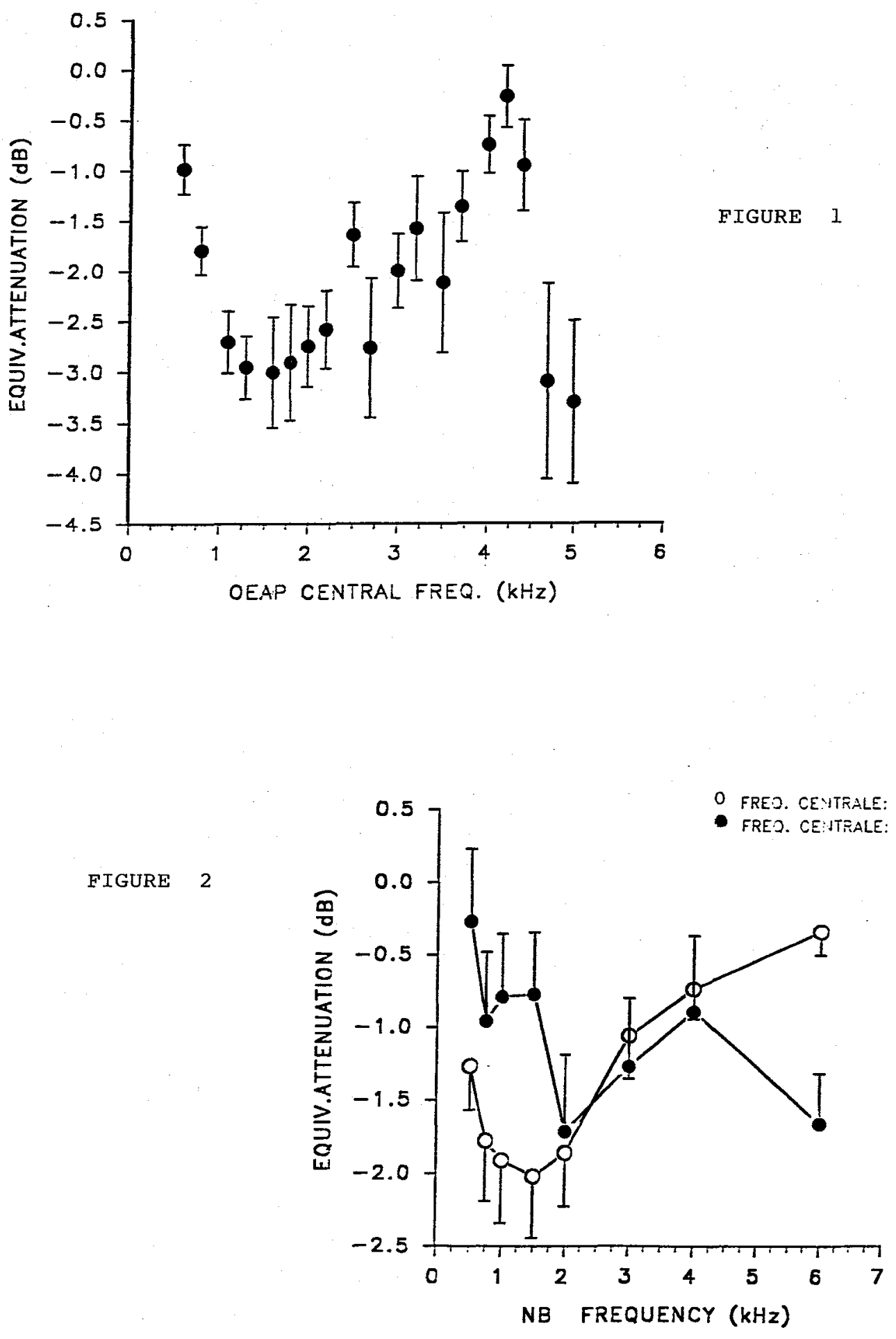